\title{
Efecto de la atención plena compasiva en dolor, ansiedad y respuestas fisiológicas en la artritis reumatoide
}

\section{Effect of compassionate mindfulness in pain, anxiety, and psychophysiological responses in rheumatoid arthritis}

\author{
Roberto Israel Patoni Maldonado ${ }^{1}$, Pedro José Rodríguez Henríquez², \\ Edgar Salinas Rivera ${ }^{3}$ y Ana Beatriz Moreno Coutiño ${ }^{1}$
}

\author{
Universidad Nacional Autónoma de México ${ }^{1}$ \\ Hospital "Dr. Manuel Gea González"2 \\ Universidad Pedagógica Nacional ${ }^{3}$
}

Autor para correspondencia: Roberto Israel Patoni Maldonado,robertronik@gmail.com.

\section{RESUMEN}

\begin{abstract}
La artritis reumatoide (AR) es una enfermedad autoinmune caracterizada principalmente por dolor, inflamación, deformidad y destrucción persistente de las articulaciones afectadas. La AR suele estar asociada con sintomatología ansiosa debido a la falta de predictibilidad de los ataques de dolor y a las complicaciones de la enfermedad. El objetivo de este proyecto fue evaluar el efecto de la atención plena compasiva (APC) en la disminución de la percepción del dolor, sintomatología ansiosa y reactividad fisiológica ante estresores de pacientes con diagnóstico de AR, ya que previamente no se había investigado el efecto de la APC sobre estas variables en México. Participaron seis pacientes del hospital "Dr. Manuel Gea González" de la Ciudad de México, los cuales asistieron a una intervención de nueve sesiones con duración de dos horas cada una, a razón de una por semana. La intervención estuvo compuesta por psicoeducación, práctica y ejercicios para el hogar. Se implementó un diseño pretest-postest con muestreo a conveniencia. Los resultados mostraron una reducción significativa de la percepción del dolor, pero no de la sintomatología ansiosa. En las reacciones fisiológicas solo se obtuvieron cambios clínicos objetivos en algunas respuestas. La intervención con APC disminuye la percepción de dolor en pacientes con AR. Se sugiere un mayor número de participantes en futuros estudios, así como seguimientos a tres, seis y doce meses.
\end{abstract}

Palabras clave: Atención plena; Compasión; Dolor; Artritis reumatoide.

\begin{abstract}
Rheumatoid arthritis (RA) is an autoimmune disease, mainly characterized by pain, inflammation, deformity, and ongoing destruction of the affected joints. RA usually generates anxiety symptoms due to the lack of predictability about ill-related pain attacks and the complications of the disease itself. Given the scarcity of Mexican studies on these problems, the present study aimed to evaluate the effect of compassion mindfulness (CM) on pain perception, anxious symptomatology, and physiological
\end{abstract}

\footnotetext{
${ }^{1}$ Facultad de Psicología, Av. Universidad 3004, Col. Copilco Universidad, Coyoacán, 04510 Ciudad de México, México, correos electrónicos: robertronik@gmail.com y moca99_99@yahoo.com.

${ }^{2}$ Calzada de Tlalpan 4800, Col. Belisario Domínguez Secc. 16, Tlalpan, 14080 Ciudad de México, México, correo electrónico: pedrorodriguez.md@gmail.com.

${ }^{3}$ Carretera Picacho-Ajusco 24, Col. Equipamiento Periférico Picacho Ajusco Canal 13, Tlalpan, 14200 Ciudad de México, México, correo electrónico: esalinas.upn@gmail.com.
} 
reactivity to stressors of patients diagnosed with RA. Six patients from a large public hospital in Mexico City received a CM intervention consisting of two-hour weekly sessions for nine weeks. The intervention protocol comprised psycho-education and practice and homework exercises, using a pre-test/post-test design through a non-probability sampling method (convenience sampling). Results showed a statistically significant reduction in pain perception but not in anxious symptomatology. Physiological reactivity decreased only partially. The authors conclude that CM reduces pain perception in RA patients. However, a larger number of participants and follow-ups at three, six, and twelve months would benefit future studies.

Key words: Mindfulness; Compassion; Pain; Rheumatoid arthritis.

Recibido: $24 / 06 / 2020$

Aceptado: 17/08/2020

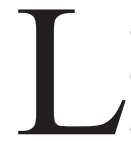

a artritis reumatoide (AR en lo sucesivo) es una enfermedad inflamatoria sistémica autoinmune que se caracteriza por dolor persistente e inflamación de las articulaciones del cuerpo, en particular las de ambas manos, pies, rodillas y tobillos, produciendo su destrucción progresiva y generando distintos grados de deformidad e incapacidad funcional. La enfermedad se asocia a la presencia de autoanticuerpos, principalmente el factor reumatoide y los anticuerpos antipéptidos cíclicos citrulinados (Anaya, 1999; Quiceno y Vinaccia, 2011). Scott, Wolfe y Huizinga (2010) explican que las células del sistema inmune, tales como los linfocitos y monocitos en un paciente con AR entran en el espacio articular y liberan sustancias químicas (enzimas, citocinas, óxido nítrico y prostaglandinas) que causan inflamación, dolor y daño a las diferentes partes de las articulaciones, como el cartílago y el hueso.

Las causas de las enfermedades reumáticas no se conocen con exactitud, pero su aparición a edades tempranas se explica por factores como la predisposición genética, lesiones, falta de actividad física, sobrepeso u obesidad, defectos de nacimiento, inflamación del recubrimiento articular, debilidad muscular, agentes ambientales e infecciosos, desequilibrio de ciertas enzimas, estrés y otros traumas emocionales (Coordinación de Ad- ministración de Riesgos Institucionales del Instituto Mexicano del Seguro Social, 2007).

En México, la AR afecta a cerca de dos millones de adultos y seis mil menores, lo que representa casi $2.2 \%$ de la población en el país (Camacho, 2017). Dicha enfermedad se manifiesta principalmente en individuos cuya edad promedio ronda los 45 años (Coordinación de Administración de Riesgos Institucionales del Instituto Mexicano del Seguro Social, 2007), aunque se ha registrado que su inicio ocurre con mayor frecuencia entre los 25 y 35 años, siendo más frecuente en el sexo femenino, a razón de cuatro mujeres por cada hombre.

El diagnóstico de AR suele ser un estresor importante debido a la incertidumbre asociada a la enfermedad, la falta de predictibilidad de los ataques de dolor y sus complicaciones. Entre los efectos a nivel laboral, social y personal están los cambios constantes de trabajo, más despidos y, por tanto, más complicaciones económicas familiares, así como un mayor aislamiento social por la estigmatización, lo que aumenta los niveles de sintomatología ansiosa, depresiva o ambas (Isik et al., 2007).

Actualmente, los tratamientos con atención plena (AP en lo sucesivo) implementados en diversos lugares del mundo han probado tener buenos resultados en la reducción de la intensidad del dolor en los diferentes padecimientos que lo conllevan, incluyendo la AR (Zhou et al., 2020). La reducción de la intensidad del dolor lo atribuyen a la práctica de no juzgar el momento presente, lo que da como resultado su aceptación, sin aumentarlo con pensamientos negativos o catastróficos (Reiner, Tibi y Lipsitz, 2013). Tang, Hölzel y Posner (2015) han propuesto tres componentes: la mejora del control atencional, una mayor capacidad de regulación emocional y una transformación de la autoconsciencia, las que interactúan entre sí para dar lugar a este beneficio.

Se ha documentado que la AP reduce la sintomatología ansiosa y los niveles de activación fisiológica asociados a la respuesta de estrés o ansiedad a través de indicadores como la frecuencia cardiaca, el cortisol y la presión sanguínea (Balconi, Fronda y Crivelli, 2019; Creswell, 2017; Pascoe, Thompson, Jenkins y Ski, 2017). 
El enlace entre compasión y AP resulta ser prometedor para aumentar el bienestar psicológico y reducir el malestar psicológico, esto lo reporta un ensayo controlado aleatorizado de Jazaieri et al. (2016) en una muestra de 100 adultos, en el que se mostró que, en comparación con un grupo en lista de espera, el programa de compasión mejoró significativamente el afecto positivo y la regulación emocional, reduciendo la divagación mental y aumentando las conductas de autocuidado. En otro estudio realizado por Chapin et al. (2014) se demostró que la meditación compasiva disminuyó el dolor y la ira en doce pacientes con dolor crónico.

En el presente estudio se reportan los efectos de la atención plena compasiva (APC), definida como "la conciencia que surge a través de prestar atención propositivamente el momento presente, sin juzgar la experiencia y generando acciones motivadas en la aspiración de liberarnos a nosotros mismos y a los demás del sufrimiento" (Moreno, 2019) en población diagnosticada con AR con dolor, sintomatología ansiosa y respuestas fisiológicas ante los estresores.

\section{MÉTODO}

\section{Participantes}

Participaron seis pacientes mujeres del hospital "Dr. Manuel Gea González" de la ciudad de México con diagnóstico de AR, con niveles de moderados a altos de dolor crónico y sintomatología ansiosa. Todas las pacientes estaban medicadas para controlar la AR y reducir el dolor, pero sin apreciar una disminución significativa del mismo. La edad promedio fue de 54.6 años (D.E. $=8$ ). Otras características sociodemográficas y médicas se muestran en la Tabla 1.

Se excluyó a pacientes con trastornos psiquiátricos, que se encontraran en otro tratamiento psicológico o con cirugía programada durante el periodo de tratamiento. En un inicio se contó con 25 participantes, divididos en un grupo control y otro experimental, pero el sismo de septiembre de 2017 damnificó a 18 de ellas, por lo que ya no pudieron asistir, y una más fue excluida por presen-
Tabla 1. Características sociodemográficas y médicas de los participantes $(n=6)$.

\begin{tabular}{|l|l|l|}
\hline \multicolumn{2}{|c|}{ Características } & N \\
\hline \multirow{4}{*}{ Escolaridad } & Primaria & 1 \\
\cline { 2 - 3 } & Secundaria & 2 \\
\cline { 2 - 3 } & Licenciatura & 3 \\
\hline \multirow{4}{*}{ Estado civil } & Soltera & 1 \\
\cline { 2 - 3 } & Unión libre & 2 \\
\cline { 2 - 3 } & Casada & 3 \\
\hline \multirow{4}{*}{ Ocupación } & Hogar & 2 \\
\cline { 2 - 3 } & Empleada & 1 \\
\cline { 2 - 3 } & Jubilada & 3 \\
\hline Comorbilidades & Síndrome de Sjögren & 1 \\
\hline \multirow{3}{*}{$\begin{array}{l}\text { Medicamentos } \\
\text { para el dolor }\end{array}$} & Paracetamol & 2 \\
\cline { 2 - 3 } & Diclofenaco & 2 \\
\cline { 2 - 3 } & Sin medicamento & 2 \\
\hline \multirow{3}{*}{$\begin{array}{l}\text { Medicamentos } \\
\text { para la ansiedad }\end{array}$} & Mirtazapina & 1 \\
\cline { 2 - 3 } & Venlafaxina & 1 \\
\cline { 2 - 3 } & Sin medicamento & 4 \\
\hline
\end{tabular}

tar rasgos de esquizofrenia, de manera que quedaron solamente seis.

\section{Materiales}

\section{Procomp Infinity}

Los indicadores o respuestas fisiológicas se registraron con ayuda del equipo Procomp Infinity para registro simultáneo de ocho canales con capacidad de $20(8 \mathrm{~Hz})$ a 2,048 $(512 \mathrm{~Hz})$ muestras por segundo, empleando para ello el software BioGraph Infinity.

Para el registro electromiográfico de superficie (EMG) se emplearon electrodos de plata-cloruro de plata $(\mathrm{Ag}, \mathrm{Ag} / \mathrm{Cl})$ colocados en la frente y en el músculo extensor del brazo derecho, con una colocación trifásica lineal a una distancia aproximada de $1 \mathrm{~cm}$ entre cada electrodo. Para la colocación de los electrodos se empleó la guía publicada por Criswell (2011); se eligieron estos grupos musculares debido a que la literatura los señala como indicadores válidos de ciertos estados emocionales, entre los que se incluyen la experiencia de estrés y la ansiedad (Arena y Schwartz, 2016; Hazlett, McLeod y Hoehn-Saric, 1994; Hoehn-Sa- 
ric, McLeod y Zimmerli, 1991). La frecuencia cardiaca se obtuvo a través del registro del volumen de flujo sanguíneo (BVP) con ayuda de un fotopletismógrafo colocado en la yema del dedo índice de la mano dominante de cada paciente. La conductancia de la piel se registró mediante dos electrodos circulares de plata-cloruro de plata colocados en la segunda falange de los dedos índice (dos) y anular (cuatro) de la mano no dominante. No se empleó ninguna solución isotónica para la instrumentación. La temperatura periférica se registró mediante de un termistor adherido a la yema del dedo medio de la mano no dominante. Antes de colocar cada sensor y electrodo se limpiaba la zona perfectamente con alcohol, o, en el caso de la conductancia de la piel, con agua. Finalmente, para el registro de la frecuencia y amplitud respiratorias se colocó una banda elástica de respiración autoadherible, compuesta por hule y velcro, a la altura del abdomen y sobre la ropa del paciente siempre y cuando esta permitiera que el sensor quedara lo suficientemente ajustado para registrar adecuadamente el movimiento del abdomen, pero no tanto como para impedir la adecuada respiración.

\section{Instrumentos}

\section{Inventario de Ansiedad de Beck (BAI).}

En su versión para México, presenta una consistencia interna de 0.83 y una confiabilidad test-retest de 0.75 (Robles et al., 2001). Clasifica la ansiedad como mínima, leve, moderada y grave según la intensidad de sus síntomas.

\section{Escala de Dolor Crónico de Lattinen (IL).}

Es una herramienta muy utilizada para la evaluación del dolor en el mundo hispanoparlante. Cuenta con una consistencia interna de $0.70 \mathrm{y}$ un coeficiente de correlación intraclase de 0.85 (Gonzalez et al., 2012). Se compone de cinco subescalas: intensidad del dolor, frecuencia del dolor, consumo de analgésicos, grado de incapacidad y horas de sueño. La puntuación de cada subescala va de 0 a 4 , dependiendo de la intensidad del dolor que percibe el paciente.

\section{Perfil de Estrés Psicofisiológico (PEP).}

Se utilizó para la medición de las respuestas fisiológicas de cada paciente a lo largo de cinco ensayos: línea base inicial, prueba de Stroop, descanso 1, recuerdo de una situación estresante y descanso 2 , con la finalidad de registrar el nivel de activación fisiológica asociada a estados alternados de estrés y relajación. La respuesta de estrés fue evocada por dos de las condiciones estresantes empleadas de manera extensa en la literatura de investigación: la prueba de Stroop y la evocación de una experiencia personal estresante (Bali y Jaggi, 2015). El PEP estuvo conformado por siete indicadores psicofisiológicos: actividad electromiográfica (EMG) de músculos frontales, actividad EMG en músculo extensor del brazo derecho, respuesta de conductancia de la piel, temperatura periférica, frecuencia cardiaca y, por último, frecuencia y amplitud respiratorias. Las instrucciones para los sujetos en cada ensayo de registro fueron las siguientes: a) Registro de línea base inicial: "Durante los próximos minutos, le pediremos que se mantenga sentada en una posición cómoda, cierre los ojos y manténgase relajada hasta que se le indique"; b) Prueba de Stroop: "Durante los siguientes minutos aparecerán en la pantalla varias listas de palabras de colores. Su tarea consistirá en decir en voz alta el color de cada palabra evitando leerla. Hágalo lo más rápido que pueda, pues las listas serán reemplazadas constantemente"; c) Descanso 1: "Durante los siguientes minutos le pediremos que permanezca lo más tranquila y relajada que pueda hasta que se le indique"; d) Recuerdo de una situación estresante: "Ahora le pediremos que piense en una situación estresante o dolorosa que haya experimentado. Enfóquese en el mayor número de detalles posibles, especialmente en los pensamientos y las emociones asociadas a la situación imaginada; e) Descanso 2: "Ahora nuevamente le pediremos que se mantenga sentada de manera cómoda y permanezca quieta y relajada con los ojos cerrados hasta que se le indique". La secuencia, los estresores y las instrucciones de cada ensayo, así como el procedimiento de instrumentación de sensores y electrodos, se tomaron directamente del estudio realizado por Salinas, Campos, Velázquez y Sánchez-Sosa (2017). 
Carta de consentimiento informado. Constó de un documento donde se informaba a las participantes de los beneficios y riesgos de su participación, así como su libertad para permanecer o no, el cual firmaban al aceptar participar en el estudio.

Audios para práctica en casa. Contenían las instrucciones para llevar a cabo en su hogar los ejercicios de atención plena revisados en las sesiones.

\section{Procedimiento}

Para medir el efecto de la APC en la percepción del dolor, la sintomatología ansiosa y el cambio de respuestas fisiológicas ante estresores en pacientes con AR, el proyecto se dividió en las cinco fases que se describen abajo.

La primera fase fue la selección de participantes, llevada a cabo por un reumatólogo experto, el cual eligió cuidadosamente a las participantes con base en su diagnóstico, historial médico, nivel de dolor y cantidad de medicamento que ingería para controlarlo, el cual no tenía efecto alguno en los niveles de dolor reportados por la paciente, para posteriormente canalizarla al psicólogo encargado del protocolo y de explicar el estudio.

En la segunda fase se dieron a conocer los riesgos y beneficios del programa; una vez que la paciente aceptaba participar se le entregaba la carta de consentimiento informado para que la firmara.

En la tercera fase se midieron los niveles de dolor con la escala de dolor crónico de Lattinen, la sintomatología ansiosa con el BAI y las respuestas fisiológicas (actividad electromiográfica frontal y del músculo extensor derecho, conductancia de la piel, frecuencia cardiaca, temperatura periférica y frecuencia y amplitud respiratorias) con el equipo Procomp Infinity ${ }^{\odot}$ con ocho canales de registro simultáneo, empleando para ello el software BioGraph Infinity en combinación con el PEP.

En la cuarta fase, un estudiante graduado, con preparación previa de seis meses en APC y practicante de técnicas de meditación, aplicó el manual de APC en su modalidad grupal, elaborado a partir de diferentes investigaciones previas basadas en los trabajos de Jon Kabat-Zinn, Matthieu

Instituto de Investigaciones Psicológicas - Universidad Veracruzana
Ricard y en la filosofía budista, consistente en los nueve módulos que se describen a continuación: 1) Psicoeducación de la atención plena y su relación con la AR. Se estableció el encuadre y una dinámica para la integración del grupo. Se expuso el concepto de estrés, la AR y sus síntomas, la relación entre las emociones y AR, el concepto y los beneficios de la AP, la compasión y la APC en el dolor y la ansiedad; 2) Bases de la atención plena compasiva. Se expuso la naturaleza de las emociones, pensamientos y sensaciones desde la perspectiva de la AP, interpretación de la realidad, emociones aflictivas y juicios internos, finalizando con una introducción a la práctica de la AP en la postura de los siete puntos; 3) La realidad del sufrimiento. Se explicó la realidad del sufrimiento, el origen y el fin de las emociones aflictivas, el concepto de desapego y las ocho actitudes de la atención plena, para terminar con la meditación sobre las acciones correctas y el amor incondicional; 4) Características de la realidad. Se mostró la influencia de la ley de causa y efecto, la impermanencia, la interdependencia y el vacío de los fenómenos aplicados a distintas experiencias de vida, concluyendo con una meditación sobre la impermanencia; 5) Práctica de la atención plena y compasión en la vida cotidiana. Se explicó cómo es posible actuar en forma automática, sin tener conciencia de la realidad, y de qué manera la práctica de la atención plena, en su forma formal o informal, conduce a estar más atento de uno mismo y del entorno; 6) Comunicación atenta y compasiva. Se expusieron los conceptos de renuncia, empatía, compasión, amor bondadoso y ecuanimidad, y la generación de relaciones compasivas consigo misma y con los demás, para finalizar con una meditación sobre la práctica del perdón; 7) Acciones que favorecen el bienestar. Se abordaron las formas de practicar la generosidad, la ética, la paciencia, el esfuerzo gozoso, la concentración y la sabiduría; 8) Atención plena compasiva aplicada a la vida cotidiana. Se detalló cómo se genera el sufrimiento en las personas y cómo este se combate a través de la comprensión de las características de la realidad, así como las acciones que generan el bienestar. 9) Mantenimiento de la práctica: Viviendo con atención plena y compasión. Se abordó la importancia de realizar un plan para la práctica constan- 
te, e identificar los obstáculos y facilitadores para esta, y terminar haciendo el cierre del tratamiento.

Los módulos fueron acompañados con audios de meditación elaborados por el laboratorio de atención plena compasiva, para que las participantes practicaran las meditaciones en casa una vez al día.

Por último, en la quinta fase se midieron los efectos del programa de APC aplicando los mismos instrumentos de la tercera etapa.

\section{Análisis estadístico}

Se vaciaron los datos en el programa estadístico SPSS, versión 19 para su análisis estadístico. Los resultados del BAI y de la escala de dolor crónico de Lattinen se analizaron mediante la prueba de Wilcoxon para determinar los efectos entre dos evaluaciones específicas cuando la variable dependiente se encuentra en el nivel ordinal. Para examinar los resultados de las respuestas fisiológicas, primero se llevó a cabo un análisis de varianza de un factor para determinar los efectos derivados de la intervención mediante la APC sobre cada respuesta fisiológica registrada. El empleo del ANOVA obedeció principalmente al nivel de medición de las variables (intervalar y de razón); el análisis comparó cada uno de los cinco ensayos del perfil de estrés antes y después del tratamiento con APC. Por último, se aplicó el índice de cambio clínico objetivo (CCO) para determinar la significancia clí- nica del efecto observado sobre cada una de las respuestas fisiológicas. El análisis se llevó a cabo con un margen de error de $5 \%$ y un nivel de confianza de $95 \%$.

\section{RESULTADOS}

\section{Percepción del dolor}

La prueba de Wilcoxon dio como resultado una disminución significativa de la percepción del dolor después del tratamiento, según la escala de dolor crónico de Lattinen $(\mathrm{z}=-2.207, p=0.027)$. La media total bajó cuatro puntos entre el pre y el postratamiento, como se puede observar en la Tabla 1.

Tabla 1. Diferencias entre medias pre y postratamiento y su probabilidad asociada $(\mathrm{n}=6)$.

\begin{tabular}{|l|c|c|c|}
\hline \multicolumn{1}{|c|}{ Variable } & Media Pre & Media Post & $\boldsymbol{p}$ \\
\hline $\begin{array}{l}\text { Percepción de dolor } \\
\text { (Puntuación IL) }\end{array}$ & 9 & 5 & $.027^{*}$ \\
\hline $\begin{array}{l}\text { Sintomatología } \\
\text { ansiosa } \\
\text { (Puntuación BAI) }\end{array}$ & 10.33 & 4.83 & .116 \\
\hline
\end{tabular}

* Efecto significativo: $p<0.05$

Todas las participantes disminuyeron su puntaje en la escala de dolor crónico de Lattinen (Figura 1), lo que significa una percepción menor del dolor crónico en todas ellas.

Figura 1. Puntuación en los niveles de dolor en el pre y postratamiento de cada participante, obtenidas con la escala de dolor crónico de Lattinen.

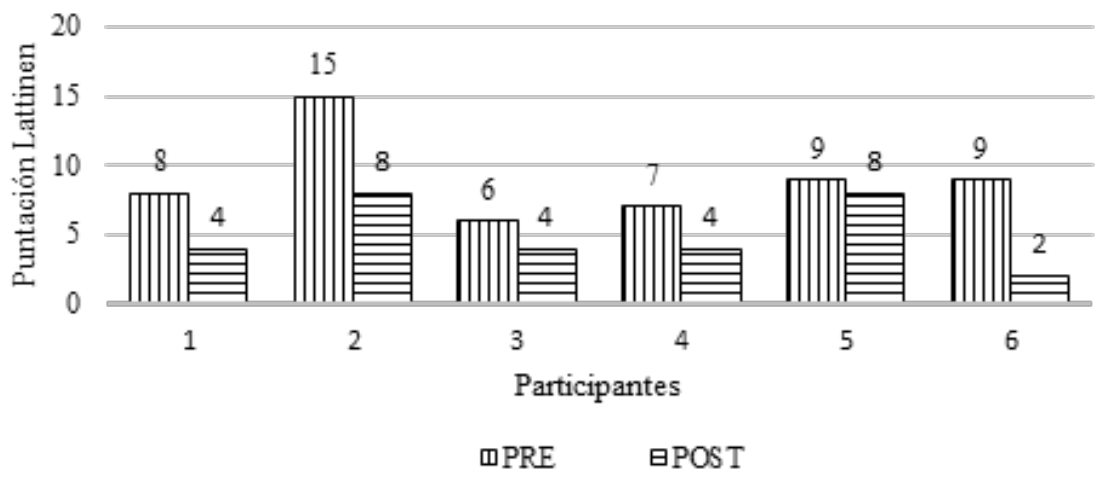




\section{Sintomatología ansiosa}

La prueba de Wilcoxon arrojó una disminución no significativa de la sintomatología ansiosa $(\mathrm{z}=-1.572$, $p=0.116)$. El puntaje de la media total disminuyó cinco puntos y medio, de acuerdo con el BAI (Tabla 1). El nivel promedio de sintomatología ansiosa en el pretratamiento fue de "leve" a "mínima" en el postratamiento, denotando una baja en la sintomatología ansiosa. Cuatro de las seis participantes disminuyeron su puntaje, y otras dos lo aumentaron de acuerdo con el BAI, tal como se puede observar en la Figura 2.

Figura 2. Puntuación y niveles pre y postratamiento de acuerdo con el BAI de cada participante.

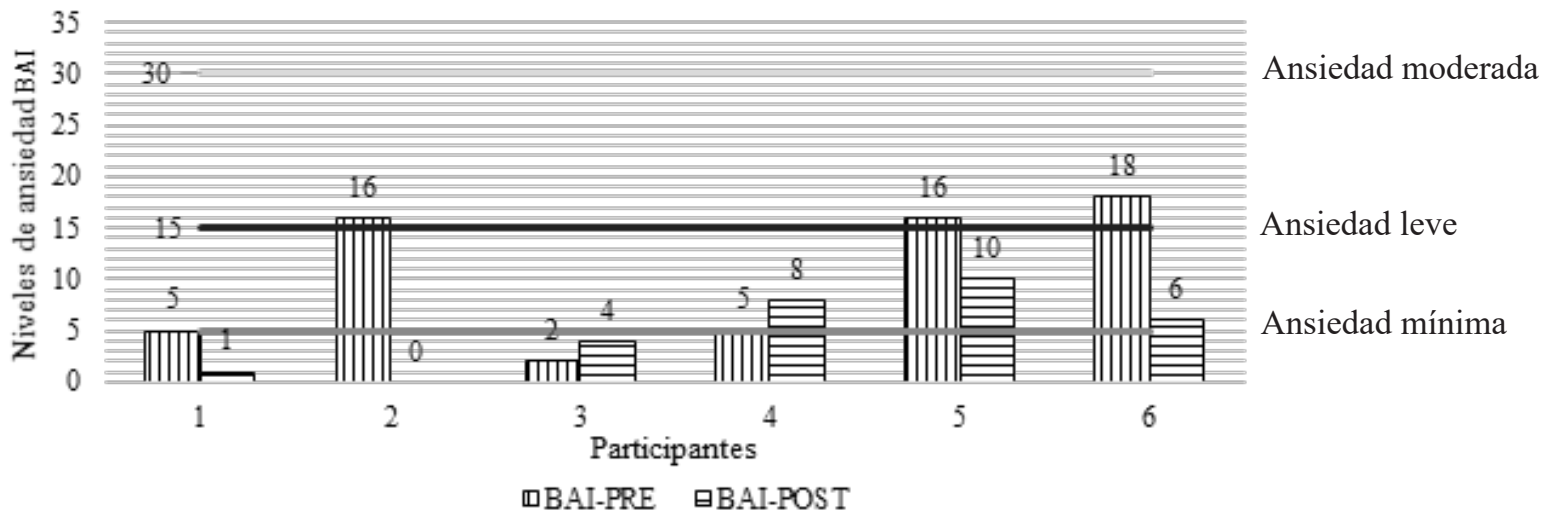

\section{Respuestas fisiológicas}

Los resultados mostrados a partir de un análisis de varianza de un factor, el cual comparó cada ensayo del PEP inicial con su medición final correspondiente a fin de determinar si las respuestas fisiológicas disminuían después de aplicar la APC, arrojó un resultado estadísticamente no significativo en los pares de las respuestas fisiológicas, excepto en el par correspondiente del EMG frontal en el ensayo de línea base inicial $(\bar{X}$ pre $=2.25 \mathrm{mV},(\bar{X}$ post $=$ $5.68, \mathrm{~F}=6.23, p=0.027)$.
En razón de los resultados no significativos mostrados por el ANOVA, se determinó realizar el índice de cambio clínico objetivo (CCO) comparando las puntuaciones medias grupales de cada indicador psicofisológico antes de la intervención con su medida grupal promedio correspondiente hacia el final de la misma (Cardiel, 1994). De acuerdo con el citado autor, las diferencias \pm .20 , es decir, $20 \%$ del cambio, reflejan efectos clínicamente significativos. Los distintos valores del CCO para cada par de comparaciones se muestran en la Tabla 2.

Tabla 2. Media total pre-post y puntajes de CCO para cada respuesta fisiológica.

\begin{tabular}{|c|c|c|c|c|c|}
\hline Respuesta & $\begin{array}{c}\text { (Media total } \\
\text { pre-post) }\end{array}$ & $\mathrm{CCO}$ & Respuesta & $\begin{array}{c}\text { (Media total } \\
\text { pre-post) }\end{array}$ & $\mathrm{CCO}$ \\
\hline \multicolumn{3}{|l|}{ a) Linea base } & \multicolumn{3}{|l|}{ d) Estresor personal } \\
\hline EMG frontal & $(2.25-5.68)$ & 1.52 & EMG frontal & $(4.38-4.22)$ & -0.03 \\
\hline EMG ext. derecho & $(3.67-3.01)$ & -0.17 & EMG ext. derecho & $(4.47-3.90)$ & -0.12 \\
\hline Conductancia piel & $(0.44-0.52)$ & 0.16 & Conductancia piel & $(0.72-1.05)$ & 0.46 \\
\hline Temp. periférica & $(32.74-34.08)$ & 0.04 & Temp. periférica & $(33.31-34.30)$ & 0.02 \\
\hline Frec. cardiaca & $(71.20-74.65)$ & 0.04 & Frec. cardiaca & $(74.37-76.28)$ & 0.02 \\
\hline Amp. respiratoria & $(1.29-1.61)$ & $0.24 *$ & Amp. respiratoria & $(1.07-1.64)$ & $0.53 *$ \\
\hline Frec. respiratoria & $(19.00-18.98)$ & -0.00 & Frec. respiratoria & $(22.16-20.75)$ & -0.06 \\
\hline
\end{tabular}




\begin{tabular}{|c|c|c|c|c|c|}
\hline \multicolumn{3}{|l|}{ b) Prueba de Stroop } & \multicolumn{3}{|l|}{ e) Descanso 2} \\
\hline EMG frontal & $(4.31-6.16)$ & 0.42 & EMG frontal & $(4.43-3.32)$ & $-0.25 *$ \\
\hline EMG ext. derecho & $(13.82-8.79)$ & $-0.36 *$ & EMG ext. derecho & $(2.08-1.71)$ & -0.18 \\
\hline Conductancia piel & $(1.06-1.31)$ & 0.24 & Conductancia piel & $(0.65-0.97)$ & 0.47 \\
\hline Temp. periférica & $(32.91-34.09)$ & 0.03 & Temp. periférica & $(33.22-34.45)$ & 0.03 \\
\hline Frec. cardiaca & $(77.66-80.79)$ & 0.04 & Frec. cardiaca & $(74.35-73.22)$ & -0.01 \\
\hline Amp. respiratoria & $(1.23-1.14)$ & -0.07 & Amp. respiratoria & (1.11-1.41) & $0.27 *$ \\
\hline Frec. respiratoria & $(32.17-33.20)$ & 0.03 & Frec. respiratoria & $(23.64-20.48)$ & -0.13 \\
\hline \multicolumn{3}{|l|}{ c) Descanso 1} & & & \\
\hline EMG frontal & $(2.12-4.14)$ & 0.94 & & & \\
\hline EMG ext. derecho & $(3.82-2.41)$ & $-0.36 *$ & & & \\
\hline Conductancia piel & $(0.71-0.96)$ & 0.35 & & & \\
\hline Temp. periférica & $(33.12-34.35)$ & 0.03 & & & \\
\hline Frec. cardiaca & $(71.61-76.01)$ & 0.06 & & & \\
\hline Amp. respiratoria & $(1.04-1.17)$ & 0.12 & & & \\
\hline Frec. respiratoria & $(28.37-29.46)$ & 0.03 & & & \\
\hline
\end{tabular}

Los números con asterisco muestran la mejoría clínica significativa o favor de la disminución de la actividad fisiológica.

Los números en negritas significan un aumento en la actividad fisiológica, la cual tiene que ser mayor a 0.20 (Cardiel, 1994).

El índice de CCO mostró una mejoría clínicamente significativa después del tratamiento de APC en la amplitud respiratoria en los ensayos de línea base, estresor personal y descanso 2 . También hubo mejora clínica en la EMG del músculo del extensor derecho en la prueba de Stroop y descanso 1, a la par en el EMG frontal en el descanso 2. Por otro lado, se incrementó la actividad en el EMG frontal en la línea base, en la prueba de Stroop y en el descanso 1. La conductancia también aumentó en la prueba de Stroop, descanso 1, estresor personal y descanso 2.

\section{DISCUSIÓN}

El presente trabajo tuvo como objetivo evaluar el efecto de la APC en la disminución de la percepción del dolor, la sintomatología ansiosa y los cambios en las respuestas fisiológicas ante diversos estresores en pacientes diagnosticadas con AR $\mathrm{y}$ dolor.

El principal hallazgo de este trabajo fue la reducción de la percepción del dolor en las seis pacientes con AR evaluadas con la escala de dolor crónico de Lattinen, mostrando una mejoría signi- ficativa en dicha variable (Wilcoxon $p=.027$ ). Tal resultado coincide con lo reportado por diferentes autores que señalan que la AP es efectiva para reducir la citada percepción (Bakhshani, Amirani, Amirifard y Shahrakipoor, 2015; Ball et al., 2017; Hilton et al., 2017; Majeed, Ali y Sudak, 2018; Zeidan y Vago, 2016), junto con complementos compasivos (Purdie y Morley, 2016; Wren et al., 2012).

En cuanto a la sintomatología ansiosa, no se logró una reducción significativa aun cuando cuatro de las seis participantes disminuyeron sus síntomas: una bajó de "moderada" a "mínima", dos de "moderada" a "leve", y una más bajó de puntuación dentro del mismo parámetro "mínimo". Por otro lado, una subió ligeramente su puntaje, pero permaneciendo en el mismo nivel mínimo, mientras que otra subió de "mínima" a "leve", quedando en parámetros no graves, estos últimos dos reportes pueden explicar que no haya habido una disminución significativa.

Respecto a las respuestas fisiológicas, en la mayoría de ellas no se encontraron diferencias significativas, lo que puede deberse a que se encontraban dentro de rangos normales al principio y al final de la intervención. Solamente hubo una 
diferencia significativa, correspondiente al EMG frontal en el ensayo de línea base, lo que está relacionado con la tensión muscular al inicio de la medición de las respuestas fisiológicas antes y después del tratamiento (Cram y Engstrom, 1986). El efecto de stairstepping (escalera) podría explicar el aumento de la tensión en el EMG frontal en línea base, ya que este fenómeno implica que suele haber reacciones excesivas en personas que tiene dificultad para relajarse ante evaluaciones y estímulos novedosos (Schwartz y Andrasik, 2003).

El análisis del CCO, mostró una mejoría clínicamente significativa en la amplitud respiratoria en los ensayos de línea base, estresor personal y descanso 2, la cual se relaciona estrechamente con estados de mayor relajación (Conde, Menéndez, Sanz y Vila, 2008). También hubo mejora clínica en la EMG del músculo del extensor derecho en la prueba de Stroop y descanso 1, y asimismo en el EMG frontal en el descanso 2, los cuales se pueden asociar con una relajación muscular más profunda (Schwartz y Andrasik, 2003). Por otro lado, se incrementó la actividad en el EMG frontal en la línea base, la prueba de Stroop y descanso 1, lo cual indica tensión muscular. La conductancia también aumentó en la prueba de Stroop, descanso 1, estresor personal y descanso 2, indicando una mayor actividad fisiológica, y por lo tanto mayor tensión. Tal incremento puede deberse a la evocación de recuerdos asociados a estados emocionales negativos (enojo, miedo, tristeza y asco), en contraste con el recuerdo de emociones asociados a estados de alegría y sorpresa (Levenson, Ekman y Friesen, 1990).

A pesar de las limitaciones de esta investigación, se alcanzó uno de los objetivos más importantes: reducir de manera estadísticamente significativa la percepción de dolor en los participantes, lo que evidencia la necesidad de abrir más espacios hospitalarios y los propios de la educación superior para el estudio de la atención plena, la compasión y las terapias cognitivo-conductuales de tercera generación, y de su efecto en el alivio de diversos padecimientos. Se sugiere una investigación más acuciosa sobre el efecto de la APC en muestras mayores y en diversas poblaciones clínicas, comparándolas con grupos de control y con seguimientos de tres, seis y doce meses, ampliando de esta manera la evidencia de su efectividad en diversos grupos clínicos de la población mexicana.

Citación: Patoni M., R.I., Rodríguez H., P.J., Salinas R., E. y Moreno C., A.B. (2022). Efecto de la atención plena compasiva en dolor, ansiedad y respuestas fisiológicas en la artritis reumatoide. Psicología y Salud,32(1), 71-80. https://doi.org/10.25009/pys.v32i1.2712.

\section{REFERENCIAS}

Anaya, J.M. (1999). Genes y artritis. Revista Colombiana de Reumatología, 6, 240-250.

Arena, J.G. y Schwartz, M.S. (2016). Introduction to pshychophysiological assessment and biofeedback baselines. En S. Schwartz y F. Andrasik (Eds.): Biofeedback: A practitioner's guide (4th ed., pp. 128-153). New York: The Guilford Press.

Bakhshani, N.M., Amirani, A., Amirifard, H. y Shahrakipoor, M. (2015). The effectiveness of mindfulness-based stress reduction on perceived pain intensity and quality of life in patients with chronic headache. Global Journal of Health Science, 8(4). Recuperado de https://doi.org/10.5539/gjhs.v8n4p142.

Balconi, M., Fronda, G. y Crivelli, D. (2019). Effects of technology-mediated mindfulness practice on stress: psychophysiological and self-report measures. Stress, 22(2), 200-209.

Bali, A. y Jaggi, A.S. (2015). Clinical experimental stress studies: Methods and assessment. Reviews in the Neurosciences, 26(5), 555-579.

Ball, E.F., Nur Shafina Muhammad Sharizan, E., Franklin, G. y Rogozińska, E. (2017). Does mindfulness meditation improve chronic pain? A systematic review. Current Opinion in Obstetrics and Gynecology. 29(6), 359-366.

Camacho S., F. (2017). Padecen artritis reumatoide 2.5\% de los mexicanos. Periódico La Jornada, miércoles 12 de julio, p. 32. Recuperado de https://www.jornada.com.mx/2017/07/12/sociedad/032n3soc.

Cardiel, R. (1994). La medición de la calidad de vida. En L. Moreno, F. Cano y H. García R. (Eds.): Epidemiología clínica (pp. 189-199). México: Interamericana-McGraw-Hill. 
Chapin, H.L., Darnall, B.D., Seppala, E.M., Doty, J.R., Hah, J.M. y Mackey, S.C. (2014). Pilot study of a compassion meditation intervention in chronic pain. Journal of Compassionate Health Care, 1(1), 4. Recuperado de https://doi.org/10.1186/ s40639-014-0004-x.

Conde P., M., Menéndez, F., Sanz M., T. y Vila A., E. (2008). The influence of respiration on biofeedback techniques. Applied Psychophysiology and Biofeedback, 33(1), 49-54.

Coordinación de Administración de Riesgos Institucionales del Instituto Mexicano del Seguro Social (2007). Evaluación de los riesgos considerados en el programa de administración de riesgos institucionales. México: IMSS. Recuperado de http:// www.imss.gob.mx/sites/all/statics/pdf/estadisticas/PARI/parievaluacion2009.pdf.

Cram, J.R. y Engstrom, D. (1986). Patterns of neuromuscular activity in pain and non-pain patients. Clinical Biofeedback \& Health: An International Journal, 9(2), 106-115.

Creswell, J.D. (2017). Mindfulness interventions. Annual Review of Psychology, 68(1), 491-516.

Criswell, E. (2011). Cram's introduction to surface electromyography. Burlington, MA: Jones \& Bartlett Learning.

Gonzalez E., J.R., Camba, A., Muriel, C., Rodriguez, M., Contreras, D. y de Barutell, C. (2012). Validación del índice de Lattinen para la evaluación del paciente con dolor crónico. Revista de La Sociedad Española del Dolor, 19(4), 181-188.

Hazlett, R.L., McLeod, D.R. y Hoehn-Saric, R. (1994). Muscle tension in generalized anxiety disroder: elevated muscle tonus or agitated movement? Psychophysiology, 31(2), 189-195.

Hilton, L., Hempel, S., Ewing, B.A., Apaydin, E., Xenakis, L., Newberry, S. y Maglione, M.A. (2017). Mindfulness meditation for chronic pain: Systematic review and meta-analysis. Annals of Behavioral Medicine, 51(2), 199-213.

Hoehn-Saric, R., McLeod, D. R. y Zimmerli, W.D. (1991). Physiological response patterns in panic disorder. Acta Psychiatrica Scandinavica, 83(1), 4-11.

Isik, A., Koca, S.S., Ozturk, A. y Mermi, O. (2007). Anxiety and depression in patients with rheumatoid arthritis. Clinical Rheumatology, 26(6), 872-878.

Jazaieri, H., Lee, I.A., McGonigal, K., Jinpa, T., Doty, J.R., Gross, J.J. y Goldin, P.R. (2016). A wandering mind is a less caring mind: Daily experience sampling during compassion meditation training. Journal of Positive Psychology, 11(1), 37-50.

Levenson, R.W., Ekman, P. y Friesen, W.V. (1990). Voluntary facial action generates emotion-specific autonomic nervous system activity. Psychophysiology, 27(4), 363-384.

Majeed, M.H., Ali, A.A., y Sudak, D.M. (2018). Mindfulness-based interventions for chronic pain: Evidence and applications. Asian Journal of Psychiatry, 32, 79-83. Doi: 10.1016/j.ajp.2017.11.025.

Moreno C., A.B. (2019). Atención plena compasiva y su estudio en México. Revista Intercontinental de Psicología y Educación, 21(1), 35-40.

Pascoe, M.C., Thompson, D.R., Jenkins, Z.M. y Ski, C.F. (2017). Mindfulness mediates the physiological markers of stress: Systematic review and meta-analysis. Journal of Psychiatric Research, 95, 156-178.

Purdie, F. y Morley, S. (2016). Compassion and chronic pain. Pain, 157(12), 2625-2627.

Quiceno, J.M. y Vinaccia, S. (2011). Artritis reumatoide: consideraciones psicobiológicas. Diversitas: Perspectivas en Psicología, 7(1), 27-41.

Reiner, K., Tibi, L. y Lipsitz, J.D. (2013). Do mindfulness-based interventions reduce pain intensity? A critical review of the literature. Pain Medicine, 2, 230-242.

Salinas R., E., Campos C., P., Velázquez J., H. y Sánchez-Sosa, J.J. (2017). Multiple-response biofeedback assisted relaxation for generalized anxiety disorder. Revista Latinoamericana de Medicina Conductual, 7(2), 1-21.

Schwartz, M.S. y Andrasik, F. (2003). Biofeedback: A practitioner's guide (3rd ed.). New York: The Guilford Press.

Scott, D.L., Wolfe, F. y Huizinga, T.W.J. (2010). Rheumatoid arthritis. The Lancet, 376(9746), 1094-1108.

Stroop, J.R. (1935). Studies of interference in serial verbal reactions. Journal of Experimental Psychology, 18(6), 643-662.

Tang, Y.Y., Hölzel, B.K. y Posner, M.I. (2015). The neuroscience of mindfulness meditation. Nature Reviews Neuroscience, 16, 213-225.

Wren, A.A., Somers, T.J., Wright, M.A., Goetz, M.C., Leary, M.R., Fras, A.M. y Keefe, F.J. (2012). Self-compassion in patients with persistent musculoskeletal pain: Relationship of self-compassion to adjustment to persistent pain. Journal of Pain and Symptom Management, 43(4), 759-770.

Zeidan, F. y Vago, D.R. (2016). Mindfulness meditation-based pain relief: a mechanistic account. Annals of the New York Academy of Sciences, 1373(1), 114-127.

Zhou, B., Wang, G., Hong, Y., Xu, S., Wang, J., Yu, H. y Yu, L. (2020). Mindfulness interventions for rheumatoid arthritis: A systematic review and meta-analysis. Complementary Therapies in Clinical Practice. Doi: 10.1016/j.ctcp.2020.101088. 